\title{
Relationship between epicardial adipose tissue and microalbuminuria in patients with newly diagnosed hypertrophic hypertension.
}

\author{
Hayati Eren ${ }^{1}$, muhammed OMAR ${ }^{2}$, ülker kaya ${ }^{3}$, Lutfi Ocal $^{4}$, mehmet inanır ${ }^{5}$, Aslı öcal $^{6}$, \\ ömer genç ${ }^{7}$, and selin genç ${ }^{8}$ \\ ${ }^{1}$ Elbistan state hospital \\ ${ }^{2}$ Fatih Sultan Mehmet Training and Research Hospital \\ ${ }^{3}$ Elbistan State Hospital \\ ${ }^{4}$ Kosuyolu Kartal Heart Taining and Research Hospital \\ ${ }^{5}$ Kosuyolu Kartal Heart Training and Research Hospital \\ ${ }^{6}$ Dr. Lütfi Kırdar Kartal Eğitim ve Araştırma Hastanesi \\ ${ }^{7}$ Kahramanmaraş Necip Fazıl City Hospital \\ ${ }^{8}$ Türkoğlu Kemal Beyazit State Hospital
}

June 8, 2020

\begin{abstract}
Background: Epicardial adipose tissue (EAT) is a cardiometabolic risk factor, and its possible relationship with hypertension has been reported previously. Microalbuminuria (MA) is associated with target-organ damage, especially in patients with hypertension that develop hypertrophy and suggest endothelial dysfunction. This study aims to investigate the relationship between echocardiographic epicardial adipose tissue (EAT) thickness and MA in patients with newly diagnosed hypertrophic hypertension. Methods: We included the newly diagnosed HT patients who applied to the outpatient clinic in a consecutive study. Patients were divided into two groups according to the presence of hypertrophy in echocardiography. We set a control group and equalized them in terms of age and gender. All subjects underwent transthoracic echocardiography to measure EAT thickness. Spot urine sample was collected for the assessment of MA. Then, appropriate statistical tests were carried out. Results: In the group with left ventricular hypertrophy $(\mathrm{LVH})$, we found EFT thicknesses and MA development rate significantly higher than the other two groups ( $5.1 \pm 1.3$ vs $5.3 \pm 1.5$ vs $6.6 \pm 1.8, \mathrm{P}<0.001$; respectively). In the group without $\mathrm{LVH}$, no relationship was found between MA and EFT. In multiple regression analyses, we determined EFT thickness (OR: 3.141 [95\% CI: 2.425-6.123], $\mathrm{P}<0.001$ ) and left ventricular mass index value (OR: 1.339 [95\% CI: 1.145-2.143], P $=0.003$ ) as independent predictors for MA development in the group with LVH. Conclusion: We think that EFT is a simple, effective and feasible method to predict target organ damage, especially in HT patients with LVH.
\end{abstract}

\section{INTRODUCTION}

Hypertension (HT) is one of the most common diseases that cause morbidity and mortality over time (1-3). It is mostly insidious (1-2). Therefore, end-organ damage has developed in many patients at the time of diagnosis, and in many of these patients, more than one end-organ damages are accompanying (1-2). Left ventricular hypertrophy $(\mathrm{LVH})$ is the mainly cardiac complication of hypertension. This condition is another manifestation of preclinical disease and has long been known as a powerful independent risk factor for all of the cardiovascular complications of hypertension and also has been associated with increased morbidity and mortality $(1,2,4)$. 
Microalbuminuria (MA) is one of the earliest marker of renal damage in patients with hypertension, and urine albumin levels are predictive for cardiovascular events in hypertensive patients (2,5-7). Parving et al. (8) have found an association between MA and essential hypertension in non-diabetic individuals for the first time. This study was supported by subsequent studies $(9,10)$. Furthermore, several studies have demonstrated that MA is an independent predictor of cardiovascular disease and increased all-cause mortality in non-diabetic as well as patients with hypertension (8-10). Development of MA in HT patients is observed, especially in patients with left ventricle hypertrophy (11-13). In other words, many patients with MA also have LVH (10-13).

Epicardial Adipose Tissue (EFT) is one of the measurements that predict and to be related to hypertrophy development in the left ventricle in HT patients, along with different imaging or laboratory methods and is related $(14,15)$. EAT is an extra-abdominal visceral fat deposit located around the heart, particularly in cardiac grooves, over the left ventricular apex and right ventricular free wall. EFT is acknowledged to be a metabolically active endocrine organ that produces several proinflammatory and proatherogenic cytokines such as angiotensinogen and free fatty acids. An association between EFT and HT has been shown in previous studies $(14,16,17)$. In addition, several previous studies have reported that EFT is associated with left ventricle hypertrophy (LVH) in hypertensive patients (14-15). Another significant point, a relationship between EFT and the presence of MA in hypertensive patients was shown (18-20). Studies suggest that both EFT and visceral adipose tissue are associated with MA (18-21). However, there is a lack of data regarding the association of epicardial fat tissue with MA in hypertensive individuals with LVH. In other words, it is observed that the group that is more associated with EFT and that the MA develops more in HT patients are LVH existing patients. In previous studies, the relationship between EFT and the development of MA in HT patients has been studied. However, the relationship between EFT and MA in patients with left ventricular hypertrophy, which has a higher risk, has not been studied. We aimed to determine the relationship between EFT and MA in newly diagnosed LVH existing HT patients.

\section{METHODS}

\section{Study population}

Patients who applied to the internal medicine and cardiology outpatient clinic between 2016 and 2019 were included in our study. Only patients who were diagnosed with new HT and had not received treatment before were included. The patients were divided into two groups as those with and without LVH according to the echocardiography. 187 patients with LVH and 173 patients without LVH were included in the study consecutively. Later, 171 normotensive individuals with age and gender equalization were determined as the control group. Patients with chronic kidney disease, diabetes mellitus (or fasting blood glucose $>125 \mathrm{mg} / \mathrm{dL}$ ), coronary heart disease, secondary causes of hypertension, previous stroke, valvular heart diseases, heart failure, atrial fibrillation, a neoplastic, inflammatory, hepatic or kidney disease (serum creatinine greater than $1.3 \mathrm{mg} / \mathrm{dl}$ in men and $1.2 \mathrm{mg} / \mathrm{dl}$ in women and positive urine culture), overt albuminuria (urinary albumin excretion $>300 \mathrm{mg} / 24 \mathrm{~h}$ ) and no available or not optimal data of the studied target organ damage) febrile condition, anemia, disabling diseases such as dementia and inability to cooperate, and poor echocardiographic quality were excluded. In addition, patients with metabolic syndrome and body mass index (BMI) $>30$ were excluded from the study. Because in these two groups; EFT would increase because of weight and decrease the effect of LVH on EFT. Finally, a total of 297 hypertensive patients and 156 control group were analyzed. All subjects underwent transthoracic echocardiography after a complete medical history and laboratory examination, including blood and spot urine samples. Patients' height, weight and blood pressure on the day of echocardiogram were recorded. The study protocol was approved by the hospital's local ethics committee in accordance with the Declaration of Helsinki and Good Clinical Practice Guidelines. Written informed consent was taken from all patients.

\section{Definition of $H T$ and the measurement method}

For new diagnosis of hypertension, office blood pressure measurements or 24-hour ambulatory blood pressure measurements were taken into consideration. In the outpatient clinic, blood pressure measurement was done 
using a mercury sphygmomanometer. The individual whose blood pressure will be measured was rested for at least ten minutes before measurement.Blood pressure was measured after asking whether the patients drank coffee or tea in last hour, or smoked in the last 30 minutes. While blood pressure was measured, the person was in a sitting position, and the arm was supported at the level of the heart and, a suitable sleeve was chosen (wrapping at least $80 \%$ of the forearm). Measurements were made from both arms, and the higher blood pressure value was taken to consideration. The diagnosis of hypertension was made by measuring systolic blood pressure value [?]140 $\mathrm{mm} \mathrm{Hg}$ and / or diastolic blood pressure value [?]90 $\mathrm{mmHg}$ in three consecutive clinical visits. Among patients whose blood pressure values were monitored on an ambulatory basis, those with average 24-hour, daytime and nighttime blood pressures values were [?] 130/80 $\mathrm{mm} \mathrm{Hg}$ [?]135/85 $\mathrm{mm}$ $\mathrm{Hg}$, and [?]120/70 $\mathrm{mmHg}$ respectively were considered as hypertensive individuals (22).

\section{Microalbuminuria}

Albuminuria was calculated by dividing albumin concentration in milligrams by creatinine concentration in grams. The presence of MA was evaluated in each patient by measuring the albumin to creatinine ratio (ACR) on three non-consecutive first morning samples as described in Pontremoli et al. (23). The mean of three non-consecutive first morning samples was recorded. Only samples from patients with negative urine cultures were collected.Urine albumin concentration was measured using a commercially available radioimmunoassay kit (Pantec, Torino, Italy). MA was defined as urine albumin/creatinine 30-300 g/mg (24). Although 24-h urine collection is still considered as the gold standard in assessing urine albumin excretion, the measurement of albumin-to-creatinine ratio in first-morning urine, preferably in two non-consecutive urine collections, has been shown to be sufficiently accurate and has been widely considered in clinical practice, also because of its low cost and feasibility (25).

\section{Echocardiography}

Two experienced cardiologists performed all the echocardiographic examinations by using Philips Affiniti 50C cardiac ultrasound scanner and 2.5-3.5 MHz transducers. In all patients, the left ventricular posterior wall thickness (PWT), interventricular septal thickness (IVST), left ventricular end-systolic diameter (LVESD), left ventricular end-diastolic diameter (LVEDD), and left atrial diameter (LAD) were measured on the parasternal long-axis view and recorded. Left ventricular ejection fractions (LVEF) of the patients were calculated by using biplane Simpson's method. Left ventricular mass (LVM) was calculated based on Devereux formula $[\mathrm{LVM}=0.8(1.04$ (IVS + LVEDD + PW) 3 - (LVEDD) 3$)+0.6]$, and body surface area was estimated using Mosteller formula [body surface area $=($ height $(\mathrm{cm}) \times$ body weight $(\mathrm{kg}) / 3600) 1 / 2$ ] $(26)$. Left ventricular mass was divided by body surface area to estimate left ventricular mass index (LVMI). LVH was defined as LVMI $>115 \mathrm{~g} / \mathrm{m}^{2}$ in males or $>95 \mathrm{~g} / \mathrm{m}^{2}$ in females (27). The coefficient of variation between the two different sonographers was $2.7 \%$, indicating the good reproducibility of the echocardiographic measurements.

\section{Epicardial adipose tissue}

Echocardiographic assessment of EAT thickness was performed as described by Iacobellis et al. (Ref). The measurement of EAT thickness was performed by TTE from a parasternal long-axis view on the right ventricle's free wall at end-diastole, and the greatest perpendicular distance to the aortic annulus was achieved and averaged over three cardiac cycles (ref). In the parasternal long-axis window, hypoechoic space on the right ventricular free wall was defined as EAT (Figure 1) (28). The intra-observer correlation coefficient was 0.956. * EAT thickness can be measured by transthoracic echocardiography, cardiac computed tomography $(\mathrm{CT})$, and cardiac magnetic resonance imaging (MRI) methods. EAT thickness measurement by echocardiography has been first validated by Iacobellis et al., who reported a good correlation between MRI and echocardiographic measurements of epicardial fat (28). An echocardiographic study found a significant correlation between epicardial fat thickness in the parasternal long- and short-axis views and LV mass $(14,28)$. If measurements by the two investigators differed by $>5 \%$ for any of the variables, the patient was excluded; if the difference was $<5 \%$, the measurements were averaged.

Other laboratory results 
Venous blood samples were obtained from each patient after an overnight fasting and a 24-hour period of abstinence from alcohol and vigorous physical exercise in order to the determination of the serum biochemical parameters. Routine serum biomarkers such as glucose, urea, creatinine, uric acid, bilirubin, alanine aminotransferase, aspartate aminotransferase, C-reactive protein, total cholesterol, high-density lipoprotein (HDL), low-density lipoprotein (LDL), triglyceride, and complete blood count were calculated by standard laboratory methods (Beckmann Coulter aU5800 Autoanalyser, Beckmann Coulter Inc, Brea, California).

\section{Statistics}

Data were analyzed using the Statistical Package for the Social Sciences, ver. 22 for Windows (SPSS, Chicago, IL). Kolmogorov-Smirnov test was used to determine the distribution properties of continuous numerical data. Comparisons among multiple groups were performed using one-way analysis of variance (ANOVA) with Tukey's post hoc test for continuous variables. Statistical analysis of numerical data that did not show normal distribution was investigated with the Kruskal-Wallis test. For analysis of categorical variables; chi-square or Fisher's exact tests were used. The correlation was tested with Spearman's analysis, where appropriate. For all statistical calculations, $\mathrm{p}<0.05$ was considered as a significant value. A logistic regression analysis was performed in order to identify any independent associates of MA in patients with LVH and without LVH, respectively. In patients with $\mathrm{LVH}$ a receiver operating characteristic (ROC) curve analysis was performed to evaluate the sensitivity, specificity, area under the curve (AUC), and confidence interval (CI) of EAT for predicting MA.

\section{RESULTS}

A total of 453 people were included in the study. Participants were divided into three groups as the control group, HT patients without left ventricular hypertrophy and HT patients with left ventricular hypertrophy. The study population included 156 healthy volunteers (mean age: $51 \pm 10$, male: 89 (57.1\%)), 154 hypertensive patients without LVH on echocardiography (mean age: $52 \pm 9$, male: $85(56.5 \%)$ ), and 143 hypertensive patients with LVH on echocardiography (mean age: $50 \pm 11$, male: 82 (57.3\%). Demographic, laboratory, and echocardiographic characteristics of the study population shown in Table 1. Age and gender distribution were similar in all groups. Routine serum biomarkers such as glucose, urea, creatinine, uric acid, alanine aminotransferase, aspartate aminotransferase, C-reactive protein, total cholesterol, HDL, LDL, triglyceride, and complete blood count parameters were similar between the groups. The values of LAD $(\mathrm{P}=0.021)$, IVST $(\mathrm{P}<0.001)$, PWT $(\mathrm{P}<0.001)$, and LVMI $(\mathrm{P}<0.001)$ were similar in the control group and the non-LVH group, but all the values were significantly higher in the patients with LVH group (Table 1). In addition, while there was no difference between the control group and the non-LVH group, in terms of EFT value, but it was significantly higher in the group with LVH compared to other groups $(5.1 \pm 1.3$ vs $5.3 \pm 1.5$ vs 6.6 $\pm 1.8, \mathrm{P}<0.001$; respectively) (Table 1 ). There was no significant difference between the groups in terms of LVEF, LVEDD and LVESD values.

According to the Pearson correlation analysis performed in the group without LVH, only a low correlation was observed between MA and DBP (Table 2). In particular, no correlation was observed with EFT (Table 2). In the group with LVH, multiple regression analysis was performed using all parameters that were significant in univariate analysis.In multivariate analysis, increased EFT (OR: 3.141 [95\% CI: 2.425-6.123], P <0.001) and increased LMVI (OR: 1.339 [95\% CI: 1.145-2.143], $\mathrm{P}=0.003$ ) was detected as independent predictors for MA development (Table 3). In ROC curve analyses, EAT thickness above $5.8 \mathrm{~mm}$ predicted the presence of MA with a sensitivity of $81.7 \%$ and a specificity of $63.6 \%$ (AUC: $797 ; 95 \%$ CI: $0.701-0.896 ; \mathrm{P}<.001$ ) (Figure 2).

\section{DISCUSSION}

In this study, we found a relationship between epicardial fat tissue (EFT) and microalbuminuria (MA) in patients with newly diagnosed essential hypertension (EH) patients with left ventricular hypertrophy (LVH). However, in our study, we did not find a relationship between the development of MA and EFT in newly diagnosed hypertension patients without LVH. We also identified EFT as an independent predictor for the presence of MA in HT patients with LVH. In other words, EFT can be used to detect early renal damage in 
patients with hypertrophy and to assess cardiovascular risk. To the best of our knowledge, this is the first study to describe this relationship.

Hypertension is a very common disease that is insidious, as it is mostly asymptomatic (1-4). Therefore, many patients have target-organ damage (TOD) in more than one organ at the time of diagnosis (2-4). The primary significance of hypertensive TOD is that it is a powerful and independent predictor of cardiovascular complications that may develop, and it usually occurs before serious events occur and predict serious complications (1-5). LVH is the major cardiac complication of hypertension and is detected by standard 12-lead electrocardiography (ECG) or transthoracic echocardiography.

Developed LVH occurs in many long-term HT patients before the onset of clinical manifestations of the disease. In addition, LVH has long been known as a strong independent risk factor for all cardiovascular complications that can develop in asymptomatic patients with hypertension and has also been associated with increased morbidity and mortality (1-4). Another important TOD in HT patients occurs in the kidney, and the first indicator of kidney damage is MA. The importance of MA, which is frequently seen in patients with essential hypertension, becomes even more important as it is an independent predictor of not only kidney damage but also high cardiovascular risk (1-4,29-31). Similarly, in our study, we found the presence of MA significantly higher in patients with HT than in the healthy population.

The most common myocardial TOD in HT patients is the ventricular hypertrophy and in kidney first MA develops as the target-organ damage. These two involvements often accompany each other. It has been shown in many studies that especially the HT patients with LVH are more associated with the development of MA, that the developments of LVH and MA in HT patients are parallel with each other, and that in many HT patients, LVH and MA develop together $(11,32,33)$. In this study, there was no significant relationship between LVMI and MA in patients non-LVH group. However, we found a positive correlation between LVMI and MA in the patients with LVH group. While there was no significant relationship between HT and MA development in some studies, it was found in some studies. The point is, the relation was found in studies involving higher stage HT patients, but in low-stage HT patients, this relationship could not be detected. Therefore, it suggests the relation between MA and LVH. We think this situation explains the results of our study (32-35). Wachtell et al. Detected MA in 1844 (23\%) of 8029 patients with stage 2-3 hypertension. In these patients, they observed that the presence of MA related to LVH regardless of age, diabetes mellitus, race, serum creatinine and smoking (2). In their study in HT patients, Yasuno et al. also detected an increase in the presence of MA as left ventricular hypertrophy increased and determined LVMI as a predictor for MA development (36). Tsioufis et al. showed that patients with MA had significantly larger left ventricular size and thicker wall thickness than patients without MA (32). The cause and effect relationship between LVH and MA is not entirely clear. It is very likely that MA is a marker of generalized increased vascular permeability, indicating universal endothelial dysfunction, increased peripheral vascular resistance due to microvascular structural and functional abnormalities, which in the same turn increases cardiac afterload leading to left ventricular hypertrophy.In addition, increasing systemic venous pressure secondary to diastolic dysfunction developing after LVH may contribute to the formation of MA by disrupting renal dynamics. Wachtell et al. suggested that angiotensin II release increased in HT and caused changes in LVH as well as increased renovascular permeability and increased MA (11). However, our study can only disclose an association between LVH and MA and does not permit any conclusions as far as the mechanism, and cause-effect relationships are concerned $(33,37,38)$. Our findings results regarding higher LVH incidence in hypertensives with high MA are consistent with previous studies (11-13).

Epicardial fat tissue (EFT) is a visceral adipose tissue located around the heart and coronary arteries and has paracrine, vasocrine and inflammatory effects (39). It has been shown to be associated with coronary artery disease, (40) metabolic syndrome, (16) insulin resistance (41) and hypertension $(15,16)$. In many recent studies, EFT has been shown to be associated with HT, and this relationship has been found to be more pronounced, especially in HT patients with LVH $(14,42,43)$. In our study, there was no difference between the control group and HT patients who did not develop LVH, whereas, in HT patients who developed LVH, we detected significantly higher EFT than these two groups. In some studies, the relation between EFT and 
HT was found, but in some other studies, no significant relationship was found between HT and EFT. The point we want to underline here is that in studies where no significant relationship was found, lower-stage HT patients were included in the study; on the contrary, in studies that found a significant relationship between EFT and HT, mostly the advanced stage HT and LVH patients included. In other words, we think that the significant relationship between HT and EFT is mostly in HT patients with LVH.

Previous studies have reported a close relationship between increased EFT and increased ventricular myocardial mass $(14,15)$. In addition, Lacobellis et al. Reported that EFT was associated with LVH rather than the presence of hypertension (14). On the other hand, increased EFT has been associated with LVH regardless of the presence of hypertension (14).

Various mechanisms may play a role in the relationship between EFT and LVH $(44,45,46)$. The exact mechanism of the increase in epicardial adipose tissue thickness and the relationship between the elevation of blood pressure and epicardial adipose tissue thickness in hypertensive patients is unknown. It is still unclear whether the increase in epicardial adipose tissue is a cause or a consequence of hypertension. To mention the possible mechanisms discussed; firstly, this mechanism may be that the elevation of the blood pressure causes the pressure load on the left ventricle and, accordingly, an increase in the thickness of the left ventricular wall, this impaired adaptation process increases the energy requirement of the myocardium and increases the mass of epicardial adipose tissue, which provides the production of free fatty acids that provide energy in the myocardium $(14,15,44)$. Another mechanism is that the increased sympathetic activity of epicardial adipose tissue via its paracrine effects leads to the development of hypertension $(44,45)$. The increase in plasma free fatty acid provided by epicardial adipose tissue may also be one of the factors that may contribute to the development of hypertension with increased sympathetic activity and impaired endothelial function by increasing plasma catecholamines $(44,45)$. It is also thought that protective adiponectins secreted from epicardial adipose tissue decrease as epicardial adipose tissue thickness increases, and this hypoadiponectinemia may result in loss of endothelial functions and arterial elastic functions (45). Another mechanism discussed is that the as the thickness of epicardial adipose tissue increases it becomes hypoxic and leads to the release of various inflammatory cytokines and vasoactive peptides (FFA, IL-6, TNF alpha, Angiotensin II, Plasma activator inhibitor I) and these released substances cause an increase in blood pressure (45-47). Similarly, in our study, we found significant correlations between EAT thickness and both LV-mass and LVMI, and the fact that we detected EFT thickness more than other groups in patients with LV hypertrophy is compatible with the current literature. In addition, autopsy series supports our data and autopsy data showed significant correlations between epicardial fat and both total heart weight and ventricular weight $(44,48)$.

Another importance of EFT is its previously shown an association with MA in different patient groups. Studies have shown that patients with MA have higher EFT (49-51). In a study on essential hypertension patients, the average EAT thickness was found higher in patients with MA than in normoalbuminuric patients (49). Akbas et al. showed that in patients with type 2 diabetes, the thickness of EAT was significantly higher in micro-albuminuric patients than in normoalbuminuric patients (50). In addition, in this study, they found that EFT is an independent marker for increased albuminuria. Similarly, higher EFT values were observed in type 1 diabetes patients with an albumin excretion rate of 30-300 mg/dL (51). Although epicardial adipose tissue is important for protective regulation of vascular functions and providing energy needs in healthy conditions, an increase in epicardial adipose tissue makes it a lipolytic, prothrombotic and proinflammatory organ (52). We have stated that EFT increases in HT patients with LVH. Increased EFT can cause advanced endothelial dysfunction and development of MA by proatherogenic, proinflammatory and bioactive molecules secreted from epicardial adipose tissue. Increased EFT reduces the production of adiponectins derived from epicardial adipose tissue, so lower adiponectin levels can accelerate the glomerular damage $(45,53)$. In our study, we did not find a significant relationship between EFT and MA in normotensive patients and HT patients without hypertrophy. However, most importantly, as one of our findings, we detected a significant relationship between EFT and MA in HT patients with hypertrophy and determined EFT as an independent predictor for the development of MA in this patient group.Our findings can be explained with the mechanisms mentioned above and, in some studies, the fact that there is no difference in terms of the presence of MA with EFT compared to normotensive patients in low-stage HT patients also supports our study $(54,55)$. 
Rather than a cause-effect relationship between MA, LVH and EFT in HT patients, these current conditions may be thought to coexist in the developing pathophysiological process in the body. Our study showed that MA is not observed in every HT patient. In particular, the group of patients with developed MA consist of patients with LVH and thicker EFT. In conclusion, we think that EFT measurements are a simple effective and applicable method to predict target organ damage, especially in patients with LVH, and maybe a useful tool to monitor the follow-up of hypertensive patients. We recommend performing a detailed renal evaluation to predict the development of kidney damage in every HT patient with thick EFT and developed LVH. We think that many studies should be done to support the results of our study.

\section{THE LIMITATIONS}

In this study, we did not study the local in tissue level or systemic markers of EAT; therefore this was an important limitation to link findings of echocardiographically measured EAT thickness and LV hypertrophy in hypertension. In our study, the "24-hour urine sample" was not studied. In the this study the presence of MA was evaluated in each patient by measuring the albumin to creatinine ratio (ACR) on three nonconsecutive first morning samples as described in Pontremoli et al. (23). However, previous studies have documented a close correlation between this ratio and measures of albuminuria from overnight or 24-hour urine collection $(24,25)$.

Other limitations of our study were its inability to measure adiponectin levels and markers of endothelial dysfunction, which may be necessary to describe the mechanisms of association between EAT and MA. Although echocardiography is a simple and inexpensive imaging method, and recent studies have shown that echocardiographic measurements are compatible with MRI measurements, echocardiographic EFT is a linear measurement from one location and may not assess the total amount of fat in epicardial tissue. In addition, patients with BMI $>30$ were excluded from the study due to the relation between EFT and overweight, in order to prevent its possible effect to results. Finally, the this study was designed to determine whether or not an association exists between albuminuria and EFT in patients with HT. However, it does not provide information on the mechanisms of this association.

CONCLUSIONMeasuring EFT thickness can help identify a high-risk group of hypertensive patients for target-organ damage. However, more extensive prospective studies involving large hypertensive populations are needed. Since MA is a well-defined cardiovascular risk marker, we think it is appropriate to use EFT, which is closely related to MA, in the cardiovascular risk classification. Depending on the results of our study, the addition of EFT measurement to routine transthoracic echocardiographic images, especially in hypertensive patients with hypertrophy, can be applied without any additional costs or time and can be used to identify patients at high risk of hypertensive complications. MA is a sign associated with subclinical organ damage and various cardiovascular risk factors in hypertension. In conclusion, we think that EFT measurements are simple, effective and feasible method to predict target organ damage, especially in patients with LVH, and maybe a useful tool to monitor the follow-up of hypertensive patients. We recommend performing a detailed renal evaluation to predict the development of kidney damage in every HT patient with thick EFT and LVH.

\section{CONFLICT OF INTERESTS}

The authors declare that there is no conflict of interests regarding the publication of this

paper.

\section{References:}

1.Tsioufis C, Stefanadis C, Toutouza M, et al. Microalbuminuria is associated withunfavourable cardiac geometric adaptations in essential hypertensive subjects. Journal of Human Hypertension. 2002;16:249-4.

2.Wachtell K, Palmieri V, Olsen $\mathrm{MH}$, et al. Urine albumin/ creatinine ratio and echocardiographic left ventricular structure and function in hypertensive patients with electrocardiographic left ventricular hypertrophy: The LIFE study. Am Heart J. 2002;143:319-26. 
3.Lieb W, Mayer B, Stritzke J, et al. Association of low-grade urinary albumin excretion with left ventricular hypertropy in the general population: The MONICA/KORA Augsburg Echocardiographic Substudy. Nephrol Dial Transplant. 2006;21:2780-7.

4.Smilde T, Asselbergs FW, Hillege HL, et al. Mild Renal Dysfunction Is Associated With Electrocardiographic Left Ventricular Hypertrophy. Am J Hypertens. 2005;18:342-347

5.Hebert LA, Spetie DN, Keane WF. The urgent call of albuminuria/proteinuria. Heeding its significance in early detection of kidney disease. Postgrad Med 2001; 110: 79-82, 87-8, 93-6.

6.Klausen K, Borch-Johnsen K, Feldt- Rasmussen B, et al. Very low levels of microalbuminuria are associated with increased risk of coronary heart disease and death independently of renal function, hypertension, and diabetes. Circulation 2004; 110: 32-5.

7.Mimran A, Ribstein J, DuCailar G. Is microalbuminuria a marker of early intrarenal vascular dysfunction in essential hypertension? Hypertension. 1994 Jun; 23(6 Pt 2): 1018-21.

8.Parving HH, Mogensen CE, Jensen HA, et al. Increased urinary albumin-excretion rate in benign essential hypertension. Lancet 1974; 1:1190 \pm 1192 .

9.Cerasola G, Cottone S, D'Ignoto G, et al. Micro-albuminuria as a predictor of cardiovascular damage in essential hypertension. J Hypertens Suppl 1989; 7:S332 \pm S333.

10.Yudkin JS, Forrest RD, Jackson CA. Microalbuminuria as predictor of vascular disease in non-diabetic

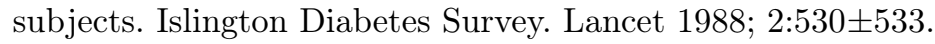

11.Wachtell K, Olsen MH, Dahlof B, et al. Microalbuminuria in hypertensive patients with electrocardiographic left ventricular hypertrophy: The LIFE study. J Hypertens 2002; 20: 405-412

12. Falqui V, Viazzi F, Leoncini G, et al. Blood pressure load, vascular permeability and target organ damage in primary hypertension. J Nephrol 2007; 20(suppl 12):63-67.

13.Salmasi AM, Jepson E, Grenfell A, et al. The degree of albuminuria is related to left ventricular hypertrophy in hypertensive diabetics and is associated with abnormal left ventricular filling: a pilot study. Angiology 2003; 54: 671-678.

14.Iacobellis G, Ribaudo MC, Zappaterreno A, et al. Relation be tween epicardial adipose tissue and left ventricular mass. Am J 2004;94:1084-7.

15.Corradi D, Maestri R, Callegari S, et al. The ventricular epicardial fat is related to the myocardial mass in normal, ischemic and hypertrophic hearts. Cardiovasc Pathol 2004;13:313-6.

16.Iacobellis G, Ribaudo MC, Assael F, et al. Echocardiographic epicardial adipose tissue is related to anthropometric and clinical parameters of metabolic syndrome: a new indicator of cardiovascular risk. J Clin Endocrinol Metab 2003;88:5163-8.

17.Ertas F, Kaya H, Acet H, et al. Increased echocardiographic epicardial fat thickness is related to impaired diurnal blood pressure profiles. Blood Press 2012;21:202-8.

18-.Ozturk MT, Ebinç FA, Okyay GU, et al. Epicardial adiposity is associated with microalbuminuria in patients with essential hypertension. Acta Cardiol. Sin. 2017, 33, 74-80.

19.Akbas, EM, Demirtas L, Ozcicek A, et al. Association of epicardial adipose tissue, neutrophil-tolymphocyte ratio and platelet-to-lymphocyte ratio with diabetic nephropathy. Int. J. Clin. Exp. Med. 2014, 7, 1794-1801.

20.Darabian S, Backlund JY, Cleary PA, et al. DCCT/EDIC Research Group. Significance of epicardial and intrathoracic adipose tissue volume among type 1 diabetes patients in the DCCT/EDIC: A pilot study. PLoS ONE 2016, 11, e0159958. 
21.Natale F, Tedesco MA, Mocerino R, et al. Visceral adiposity and arterial stiffness: echocardiographic epicardial fat thickness reflects, better than waist circumference, carotid arterial stiffness in a large population of hypertensives. Eur J Echocardiogr 2009; 10:549-55

22.Chobanian AV, Bakris GL, Black HR, et al. Seventh report of the Joint National Committee on Prevention, Detection, Evaluation, and Treatment of High Blood Pressure. Hypertension 2003;42:1206-52.

23.Pontremoli R, Sofia A, Raveraet M, et al. Prevalence and clinical correlates of microalbuminuria in essential hypertension. The MAGIC study. Hypertension 1997; 30: 1135-1143.

24. Mattix HJ, Hsu, C, Shaykevich S, et al. Use of the Albumin/Creatinine Ratio to Detect Microalbuminuria (2002): Implications of Sex and Race. Journal of the American Society of Nephrology, 13, 1034-1039.

25.Viazzi F, Leoncini G, Pontremoli R. Global cardiovascular risk assessment in the management of primary hypertension: the role of the kidney. Int J Hypertens 2013:542646. 26.Devereux RB, Alonso DR, Lutas EM, et al. Echocardiographic assessment of left ventricular hypertrophy: comparison to necropsy findings. Am J Cardiol. 1986;57:450 - 458.

27.Lang RM, Badano LP, Mor-Avi V, et al. Recommendations for cardiac chamber quantification by echocardiography in adults: an update from the American Society of Echocardiography and the European Association of Cardiovascular Imaging. Eur Heart J Cardiovasc Imaging. 2015 Mar; 16(3): 233-70.

28.Iacobellis G, Assael F, Ribaudo MC, et al. Epicardial fat from echocardiography: a new method for visceral adipose tissue prediction. Obes Res 2003; 11:304-310.

29. Ljungman S, Wilkstrand J, Hartford M, et al.Urinary albümin excretions - a predictor of risk of cardiovascular disease. A prospective 10-year follow-up of middle aged non diabetic normal and hypertensive men. Am J Hypertens 1996;9:770 -778.

30.Bigazzi R, Bianchi S, Baldari D, et al. Microalbuminuria predicts cardiovascular and renal insufficiency in patients with essential hypertension. J Hypertens 1998;16:1325-1333.

31. Pontremoli R, Viazzi F, Sofia A, et al. Microalbuminuria: a marker of cardiovascular risk and organ damage in essential hypertension. Kidney Int 1997;52(suppl 63):S-163- S-165.

32. Smilde T, Asselbergs FW, Hillege HL, et al. Mild Renal Dysfunction Is Associated With Electrocardiographic Left Ventricular Hypertrophy. Am J Hypertens. 2005;18:342-347.

33.Tsioufis C, Dimitriadis K, Selima M, et al. Association of resistin with urinary albumin excretion in nondiabetic patients with essential hypertension. Am J Hypertens 2010; 23:681-686.

34.Albuquerque FN, Somers VK, Blume G, et al. Usefulness of epicardial adipose tissue as predictor of cardiovascular events in patients with coronary artery disease. Am. J. Cardiol. 2012, 110, 1100-1105.

35.Shim IK, Cho KI, Kim HS, et al. Impact of Gender on the Association of Epicardial Fat

Thickness, Obesity, and Circadian Blood Pressure Pattern in Hypertensive Patients. J. Diabetes Res. $2015 ; 2015: 924539$

36. Yasuno S, Ueshima K, Oba K, et al. Clinical significance of left ventricular hypertrophy and changes in left ventricular mass in high risk hypertensive patients: a subanalysis of the Candesartan Antihypertensive Survival Evaluation in Japan trial. J Hypertens 2009; 27:1705-1712.

37. Tsioufis C, Dimitriadis K, Andrikou E, et al. ADMA, C-reactive protein, and albuminuria in untreated essential hypertension: a cross-sectional study. Am J Kidney Dis 2010; 55:1050-1059.

38. Stehouwer CD, Smulders YM. Microalbuminuria and risk for cardiovascular disease: Analysis of potential mechanisms. J Am Soc Nephrol 2006; 17: 2106-2111. 
39. Iacobellis G, Corradi D, Sharma AM. Epicardial adipose tissue: anatomic, biomolecular and clinical relationships with the heart. Nat Clin Pract Cardiovasc Med 2005;2:536-43.

40. Ahn SG, Lim HS, Joe DY, et al.bRelationship of epicardial adipose tissue by echocardiography to coronary artery disease. Heart 2008;94:e7.

41. Iacobellis G, Leonetti F. Epicardial adipose tissue and insülin resistance in obese subjects. J Clin Endocrinol Metab 2005;90:6300-2.

42. Eroğlu S, Sade LE, Yıldırır A, et al. Association of epicardial adipose tissue thickness by echocardiography and hypertension. Turk Kardiyol Dern Ars 2013;41:115-22.

43. Dicker D, Atar E, Kornowski R, et al. Increased epicardial adipose tissue thickness as a predictor for hypertension: a cross-sectional observational study. J Clin Hypertens (Greenwich) 2013;15:893-8.

44. Womack HC. The relationship between human body weight, subcutaneous fat, heart weight, and epicardial fat. Hum Biol.1983;55:667-76.

45. Teijeira-Fernandez E, Eiras S, Grigorian-Shamagian L, et al. Epicardial adipose tissue expression of adiponectin is lower in patients with hypertension. J Hum Hypertens 2008;22:856-63.

46. Iacobellis G, Barbaro G. The double role of epicardial adipose tissue as pro- and anti-inflammatory organ. Horm Metab Res 2008;40:442-5.

47. Sacks HS, Fain JN. Human epicardial fat: what is new and what is missing? Clin Exp Pharmacol Physiol 2011;38:879-87.

48. Sons HU, Hoffmann V. Epicardial fat cell size, fat distribution and fat infiltration of the right and left ventricle of the heart. Anat Anz 1986;161:355-73.

49. Ozturk MT, Ebinç FA, Okyay GU, et al. Epicardial adiposity is associated with microalbuminuria in patients with essential hypertension. Acta Cardiol. Sin. 2017, 33, 74-80.

50. Akbas EM, Demirtas L, Ozcicek A, et al. Association of epicardial adipose tissue, neutrophil-tolymphocyte ratio and platelet-to-lymphocyte ratio with diabetic nephropathy. Int. J. Clin. Exp. Med. 2014, 7, 1794-1801.

51. Darabian S, Backlund JY, Cleary PA, et al. DCCT/EDIC Research Group. Significance of epicardial and intrathoracic adipose tissue volume among type 1 diabetes patients in the DCCT/EDIC: A pilot study. PLoS ONE 2016, 11, e0159958.

52. Iozzo P. Myocardial, perivascular, and epicardial fat. Diabetes Care 2011;34 Suppl 2:S371-9.

53.Sharma K, Ramachandrarao S, Qiu G, et al. Adinopectin regulates albuminuria and podocyte function in mice. J Clin Invest 2008; 118:1645-56.

54.Palatini $\mathrm{P}$ et al. Target-organ damage in stage I hypertensive subjects with white coat and sustained hypertension: results from the HARVEST study. Hypertension 1998; 31: 57-63.

55.Hoegholm A, Kristensen KS, Bang LE, et al. White coat hypertension and target organ involvement: the impact of different cut-off levels on albuminuria and left ventricular mass and geometry. J Hum Hypertens 1998; 12: 433-439.

\section{FIGURE LEGENDS:}

Figure 1: Transthoracic echocardiography parasternal long-axis view demonstrating the epicardial adipose tissue as the hypoechoic space on the right ventricular free wall at end-diastole.

Figure 2: The receiver operating curve analysis provided that an epicardial adipose tissue thickness greater than $5.8 \mathrm{~mm}$ predicted the MA with a sensitivity of $81.7 \%$ and a specificity of $63.6 \%(\mathrm{P}<.001)$. 
Table 1: Comparison of the demographic, laboratory, and echocardiographic characteristics of the study population.

\begin{tabular}{|c|c|c|c|c|}
\hline Variables & Control (n:156) & Non-LVH group (n:154) & With LVH group (n:143) & $\mathrm{P}$ value \\
\hline \multicolumn{5}{|l|}{ Baseline demographics } \\
\hline Age, years & $51 \pm 10$ & $52 \pm 9$ & $50 \pm 11$ & 0,712 \\
\hline Gender, male (n, \%) & $89(57.1)$ & $87(56.5)$ & $82(57.3)$ & 0.655 \\
\hline Dyslipidemia (n, \%) & $43(27.6)$ & $41(26.6)$ & $38(31.7)$ & 0.435 \\
\hline Smoking $(\mathrm{n}, \%)$ & $48(30.8)$ & $48(31.2)$ & $48(33.6)$ & 0.633 \\
\hline BMI $(\mathrm{kg} / \mathrm{m} 2)$ & $26.9 \pm 3.3$ & $27.1 \pm 3.6$ & $27.2 \pm 3.8$ & .748 \\
\hline $\mathrm{SBP}(\mathrm{mm} \mathrm{Hg})$ & $119 \pm 8$ & $145 \pm 13^{*}$ & $165 \pm 18^{*,+}$ & $<0.001$ \\
\hline $\mathrm{DBP}(\mathrm{mm} \mathrm{Hg})$ & $78 \pm 7$ & $96 \pm 12^{*}$ & $103 \pm 10^{*},+$ & $<0.001$ \\
\hline Heart rate (pbm) & $74 \pm 13$ & $75 \pm 14$ & $76 \pm 12$ & 0.611 \\
\hline \multicolumn{5}{|l|}{ Laboratory } \\
\hline MA $(n, \%)$ & $5(3.2)$ & $30(20.1)^{*}$ & $59(41.2)^{*},+$ & $<0.001$ \\
\hline Fasting blood glucose $(\mathrm{mg} / \mathrm{dL})$ & $97 \pm 18$ & $96 \pm 16$ & $99 \pm 19$ & 0.322 \\
\hline Creatinine $(\mathrm{mg} / \mathrm{dL})$ & $0.71 \pm 0.22$ & $0.74 \pm 0.24$ & $0.75 \pm 0.23$ & 0.412 \\
\hline $\mathrm{BUN}(\mathrm{mg} / \mathrm{dl})$ & $14 \pm 3$ & $16 \pm 3$ & $16 \pm 4$ & 0.376 \\
\hline Uric acid (mg/dL) & $4.81 \pm 1.56$ & $4.96 \pm 1.19$ & $4.99 \pm 1.21$ & 0.543 \\
\hline Hemoglobin (g/dL) & $14.2 \pm 1.7$ & $14.6 \pm 1.3$ & $14.4 \pm 1.5$ & 0,255 \\
\hline $\mathrm{WBC}(\mathrm{x} 103 / \mathrm{mL})$ & $7.34 \pm 1.64$ & $7.25 \pm 1.69$ & $7.11 \pm 1.57$ & 0.771 \\
\hline Platelet (x103cells/dL) & $250 \pm 45$ & $252 \pm 43$ & $244 \pm 51$ & 0,322 \\
\hline $\operatorname{AST}(\mathrm{U} / \mathrm{L})$ & $34 \pm 8$ & $35 \pm 7$ & $36 \pm 9$ & 0.656 \\
\hline $\operatorname{ALT}(\mathrm{U} / \mathrm{L})$ & $23 \pm 6$ & $22 \pm 5$ & $25 \pm 7$ & 0.234 \\
\hline $\mathrm{LDL}(\mathrm{mg} / \mathrm{dL})$ & $132 \pm 38$ & $130 \pm 36$ & $133 \pm 37$ & 0.811 \\
\hline $\mathrm{HDL}(\mathrm{mg} / \mathrm{dL})$ & $45 \pm 13$ & $44 \pm 12$ & $43 \pm 13$ & 0.242 \\
\hline Triglycerides $(\mathrm{mg} / \mathrm{dL})$ & $146 \pm 43$ & $142 \pm 41$ & $149 \pm 47$ & 0.141 \\
\hline Total cholesterol $(\mathrm{mg} / \mathrm{dL})$ & $211 \pm 44$ & $207 \pm 40$ & $214 \pm 45$ & 0.242 \\
\hline $\mathrm{CRP}(\mathrm{mg} / \mathrm{dL})$ & $4.4 \pm 1.2$ & $4.2 \pm 1.1$ & $4.5 \pm 1.4$ & 0.645 \\
\hline \multicolumn{5}{|l|}{ Echocardiography } \\
\hline LVEF, $(\%)$ & $63 \pm 3$ & $63 \pm 2$ & $65 \pm 4$ & 0.167 \\
\hline $\mathrm{LAD},(\mathrm{mm})$ & $35 \pm 3$ & $36 \pm 2$ & $38 \pm 3^{*,+}$ & 0.021 \\
\hline LVEDD, $(\mathrm{mm})$ & $44.7 \pm 6.2$ & $44.6 \pm 5.9$ & $44.4 \pm 5.8$ & 0.167 \\
\hline LVESD, (mm) & $29.2 \pm 4.5$ & $29.0 \pm 4.4$ & $28.9 \pm 4.2$ & 0.143 \\
\hline IVST, $(\mathrm{mm})$ & $96.6 \pm 1.5$ & $100.6 \pm 1.4$ & $12.8 \pm 2.1^{*,+}$ & $<0.001$ \\
\hline PWT, (mm) & $94.9 \pm 1.2$ & $98.8 \pm 1.3$ & $12.6 \pm 1.9^{*},+$ & $<0.001$ \\
\hline LVMI, (gr/m2) & $84.2 \pm 21.4$ & $90.6 \pm 24.1$ & $118.3 \pm 34.5^{*},+$ & $<0.001$ \\
\hline EAT thickness, (mm) & $5.1 \pm 1.3$ & $5.3 \pm 1.5$ & $6.6 \pm 1.8^{*},+$ & $<0.001$ \\
\hline
\end{tabular}

${ }^{*} \mathrm{p}<0.05$ vs. control group; ${ }^{+} \mathrm{p}<0.05$ vs. without LVH group;

Abbreviations: ALT, alanine aminotransferase; AST, aspartate aminotransferase; BMI, body mass index; BUN, blood urea nitrogen; CRP, C-reactive protein;DBP, diastolic blood pressure; EAT, epicardial adipose tissue; HDL, high density lipoprotein; HT, hypertension; IVST, interventricular septum thickness; MA, microalbuminuria; LAD, left atrial diameter;LDL, low-density lipoprotein; LVCR, left ventricular constrictive remodeling; LVEDD, left ventricular end diastolic diameter; LVEF, left ventricular ejection fraction; LVESD, left ventricular end systolic diameter; LVH, left ventricular hypertrophy; LVM, left ventricular mass; LVMI, left ventricular mass index;PWT, posterior wall thickness; SBP, systolic blood pressure. Continuous variables with normal distribution were expressed as mean $\pm \mathrm{SD}$ and continuous variables without normal distribution were expressed as median (25th-75th percentiles). 
Table 2. Pearson correlation analysis between MA and other variables in HT patients without LVH.

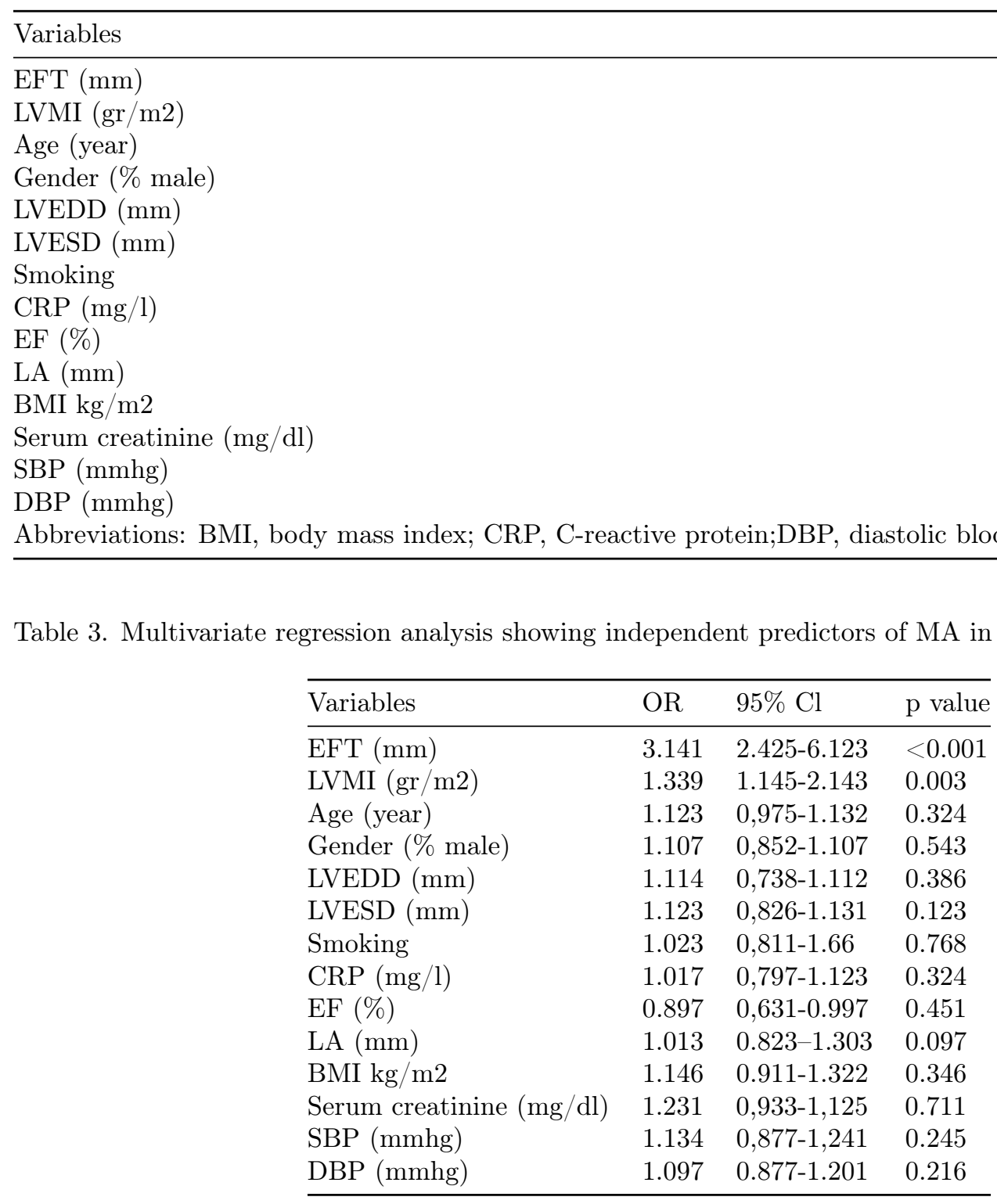

Abbreviations: BMI, body mass index; CRP, C-reactive protein;DBP, diastolic blood pressure; EAT, epicardial adipose tissue; MA, microalbuminuria; LAD, left atrial diameter; LVEDD, left ventricular end diastolic diameter; LVEF, left ventricular ejection fraction; LVESD, left ventricular end systolic diameter;LVMI, left ventricular mass index; SBP, systolic blood pressure; LVH, left ventricular hypertrophy; 

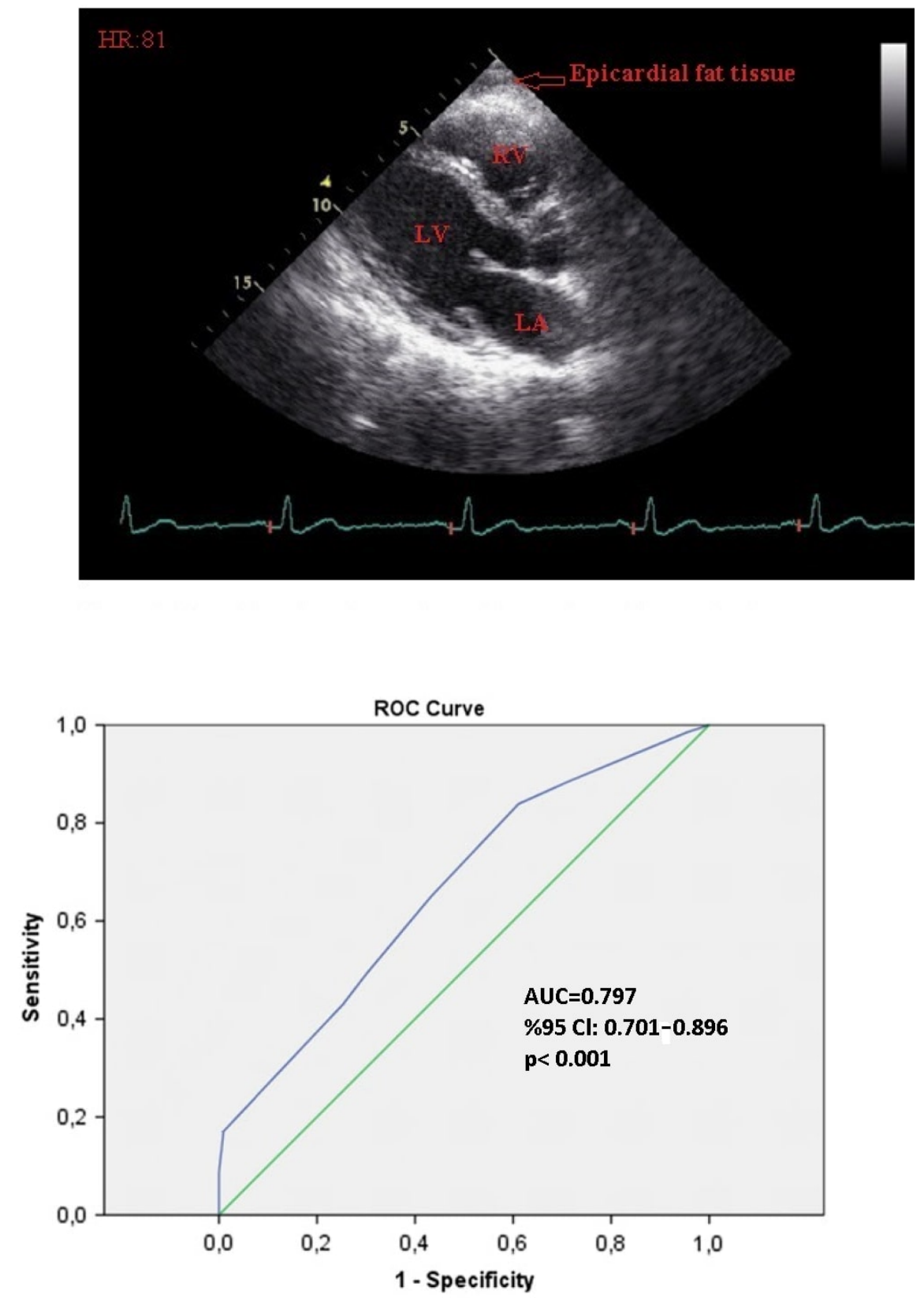